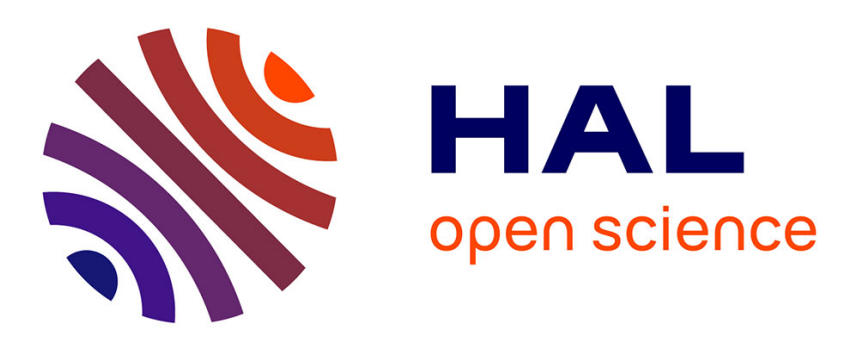

\title{
A characterization of those automata that structurally generate finite groups
}

Ines Klimann, Matthieu Picantin

\section{To cite this version:}

Ines Klimann, Matthieu Picantin. A characterization of those automata that structurally generate finite groups. 11th Latin American Theoretical INformatics Symposium (LATIN 2014), 2014, Uruguay. pp.180-189. hal-00877087

\section{HAL Id: hal-00877087 \\ https: / hal-univ-paris.archives-ouvertes.fr/hal-00877087}

Submitted on 25 Oct 2013

HAL is a multi-disciplinary open access archive for the deposit and dissemination of scientific research documents, whether they are published or not. The documents may come from teaching and research institutions in France or abroad, or from public or private research centers.
L'archive ouverte pluridisciplinaire HAL, est destinée au dépôt et à la diffusion de documents scientifiques de niveau recherche, publiés ou non, émanant des établissements d'enseignement et de recherche français ou étrangers, des laboratoires publics ou privés. 


\title{
A Characterization of those Automata that Structurally Generate Finite Groups
}

\author{
Ines Klimann^ and Matthieu Picantin^ \\ Univ Paris Diderot, Sorbonne Paris Cité, LIAFA, \\ UMR 7089 CNRS, F-75013 Paris, France \\ \{klimann, picantin\}@liafa. univ-paris-diderot.fr
}

\begin{abstract}
Antonenko and Russyev independently have shown that any Mealy automaton with no cycles with exit - that is, where every cycle in the underlying directed graph is a sink component-generates a finite (semi)group, regardless of the choice of the production functions. Antonenko has proved that this constitutes a characterization in the non-invertible case and asked for the invertible case, which is proved in this paper.
\end{abstract}

Keywords: automaton groups, Mealy automata, finiteness problem

\section{Introduction}

The class of automata (semi)groups contains multiple interesting and complicated (semi)groups with sometimes unusual features [4].

In the last decades, the classical decision problems have been investigated for such (semi)groups. The word problem is solvable using standard minimization techniques, while the conjugacy problem is undecidable for automata groups [22]. Of special interest for our concern here, the finiteness problem was proved to be undecidable for automata semigroups [9] and remains open for automata groups despite several positive and promising results $[1,2,5,6,12-15,19,20]$.

The family of automata with no cycles with exit was investigated by Antonenko and by Russyev independently. Focused on the invertible case, Russyev stated in [17] that any invertible Mealy automata with no cycles with exit generates a finite group. Meanwhile, Antonenko showed in [2] (see also [3]) the same result in the non-invertible case and proved the following maximality result: for any automaton with at least one cycle with exit, it is possible to choose (highly non-invertible) production functions

\footnotetext{
* Both authors are partially supported by the french Agence Nationale pour la Recherche, through the Project MealyM ANR-JCJC-12-JS02-012-01.
} 
such that the semigroup generated by the induced Mealy automaton is infinite.

In this paper, we fill the visible gap by extending the aforesaid maximality result to the invertible case: for any automaton with at least one cycle with exit, it is possible to choose invertible production functions such that the group generated by the induced Mealy automaton is infinite.

The proof of this new result makes use of original arguments for the current framework, whose common idea is to put a special emphasis on the dual automaton, obtained by exchanging the roles of the stateset and the alphabet. Thereby it continues to validate the general strategy first suggested in the paper [1], then followed and continuously developed in $[12,13]$.

The new maximality result provides a precious milestone in the ongoing work by De Felice and Nicaud (see [7] for a first paper) who propose to design random generators for finite groups based on those invertible Mealy automata with no cycles with exit. Their aim is to simulate interesting distributions that might offer a wide diversity of different finite groups by trying to avoid the classical concentration phenomenon around a typical object, namely symmetric or alternating groups $[8,11]$, which is significant in already studied distributions. Once implemented, such generators would be very useful to test the performance and robustness of algorithms from computational group theory. They would also be of great use when trying to check a conjecture, by testing it on various random inputs, since exhaustive tests are impossible due to a combinatorial explosion.

The structure of the paper is the following. Basic notions on Mealy automata and automaton (semi)groups are presented in Section 2. In Section 3 , we introduce new tools and prove the main result.

\section{Mealy Automata}

This section contains material for the proofs: first classical definitions and then considerations already made in [12] to maintain the paper selfcontained.

\subsection{Automaton Groups and Semigroups}

If one forgets initial and final states, a (finite, deterministic, and complete) automaton $\mathcal{A}$ is a triple

$$
\left(A, \Sigma, \delta=\left(\delta_{i}: A \rightarrow A\right)_{i \in \Sigma}\right),
$$


where the stateset $A$ and the alphabet $\Sigma$ are non-empty finite sets, and where the $\delta_{i}$ are functions called transition functions.

The transitions of such an automaton are

$$
x \stackrel{i}{\longrightarrow} \delta_{i}(x) .
$$

An automaton is reversible if all its transition functions are permutations of the stateset. Note that in this case each state has exactly one incoming transition labelled by each letter.

A Mealy automaton is a quadruple

$$
\left(A, \Sigma, \delta=\left(\delta_{i}: A \rightarrow A\right)_{i \in \Sigma}, \rho=\left(\rho_{x}: \Sigma \rightarrow \Sigma\right)_{x \in A}\right),
$$

such that both $(A, \Sigma, \delta)$ and $(\Sigma, A, \rho)$ are automata. In other terms, a Mealy automaton is a letter-to-letter transducer with the same input and output alphabet. If $\mathcal{A}=(A, \Sigma, \delta)$ is an automaton and $\rho=\left(\rho_{x}: \Sigma \rightarrow\right.$ $\Sigma)_{x \in A}$ is a finite sequence of functions, we denote by $(\mathcal{A}, \rho)$ the Mealy automaton $(A, \Sigma, \delta, \rho)$ and we say that $\mathcal{A}$ is enriched with $\rho$. The graphical representation of a Mealy automaton is standard, see Fig. 1.

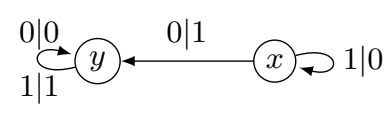

Fig. 1. An example of a Mealy automaton: the so-called adding machine.

The transitions of a Mealy automaton are

$$
x \stackrel{i \mid \rho_{x}(i)}{\longrightarrow} \delta_{i}(x) \text {. }
$$

A Mealy automaton $\mathcal{M}=(A, \Sigma, \delta, \rho)$ is reversible if the automaton $(A, \Sigma, \delta)$ is reversible and invertible if the functions $\rho_{x}$ are permutations of the alphabet. In this latter case, its inverse is the Mealy automaton $\mathcal{M}^{-1}$ with stateset $A^{-1}=\left\{x^{-1}, x \in A\right\}$ and set of transitions

$$
x^{-1} \stackrel{j \mid i}{\longrightarrow} y^{-1} \in \mathcal{M}^{-1} \quad \Longleftrightarrow \quad x \stackrel{i \mid j}{\longrightarrow} y \in \mathcal{M} .
$$

A Mealy automaton $\mathcal{M}$ is bireversible if both $\mathcal{M}$ and $\mathcal{M}^{-1}$ are invertible and reversible.

In a Mealy automaton $\mathcal{M}=(A, \Sigma, \delta, \rho)$, the sets $A$ and $\Sigma$ play dual roles. So we may consider the dual (Mealy) automaton defined by

$$
\mathfrak{o}(\mathcal{M})=(\Sigma, A, \rho, \delta)
$$




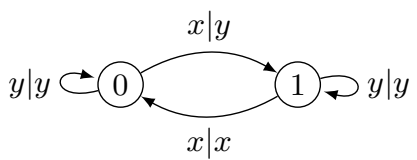

Fig. 2. The dual automaton of the Mealy automaton of Fig. 1.

see an example on Fig. 2. Obviously, a Mealy automaton is reversible if and only if its dual is invertible.

Let $\mathcal{M}=(A, \Sigma, \delta, \rho)$ be a Mealy automaton. We view $\mathcal{M}$ as an automaton with an input and an output tape, thus defining mappings from input words over $\Sigma$ to output words over $\Sigma$. Formally, for $x \in A$, the map $\rho_{x}: \Sigma^{*} \rightarrow \Sigma^{*}$, extending $\rho_{x}: \Sigma \rightarrow \Sigma$, is defined by:

$$
\forall i \in \Sigma, \forall \mathbf{s} \in \Sigma^{*}, \quad \rho_{x}(i \mathbf{s})=\rho_{x}(i) \rho_{\delta_{i}(x)}(\mathbf{s}) .
$$

By convention, the image of the empty word is itself. The mapping $\rho_{x}$ is length-preserving and prefix-preserving. We say that $\rho_{x}$ is the production function associated with $x$ in $\mathcal{M}$ or, more briefly, if there is no ambiguity, the production function of $x$. For $\mathbf{u}=x_{1} \cdots x_{n} \in A^{n}$ with $n>0$, we set $\rho_{\mathbf{u}}: \Sigma^{*} \rightarrow \Sigma^{*}, \rho_{\mathbf{u}}=\rho_{x_{n}} \circ \cdots \circ \rho_{x_{1}}$.

Denote dually by $\delta_{i}: A^{*} \rightarrow A^{*}, i \in \Sigma$, the production functions associated with the dual automaton $\mathfrak{d}(\mathcal{M})$. For $\mathbf{s}=i_{1} \cdots i_{n} \in \Sigma^{n}$ with $n>0$, we set $\delta_{\mathrm{s}}: A^{*} \rightarrow A^{*}, \delta_{\mathrm{s}}=\delta_{i_{n}} \circ \cdots \circ \delta_{i_{1}}$.

The semigroup of mappings from $\Sigma^{*}$ to $\Sigma^{*}$ generated by $\rho_{x}, x \in A$, is called the semigroup generated by $\mathcal{M}$ and is denoted by $\langle\mathcal{M}\rangle_{+}$. When $\mathcal{M}$ is invertible, its production functions are permutations on words of the same length and thus we may consider the group of mappings from $\Sigma^{*}$ to $\Sigma^{*}$ generated by $\rho_{x}, x \in A$; it is called the group generated by $\mathcal{M}$ and is denoted by $\langle\mathcal{M}\rangle$.

The automaton of Fig. 1 generates the semigroup $\mathbb{N}$ and the group $\mathbb{Z}$. The orbit of the word $0^{n}$ under the action of $\rho_{x}$ is of size $2^{n}$ : it acts like a binary addition until $1^{n}$ (considering the most significant bit on the right). In fact the Mealy automaton of Fig. 1 is called the adding machine [10].

Remind some known facts on finiteness of the automaton (semi)group:

(F1) To prune a Mealy automaton by deleting its states which are not reachable from a cycle (see the precise definition of a cycle in Subsection 3.1) does not change the finiteness or infiniteness of the generated (semi)group. 
(F2) An invertible Mealy automaton generates a finite group if and only if it generates a finite semigroup [1,21].

(F3) A Mealy automaton generates a finite semigroup if and only if so does its dual $[1,16,18]$.

(F4) An invertible-reversible but not bireversible Mealy automaton generates an infinite group [1].

Whenever the alphabet is unary, the generated group is trivial and there is nothing to say. Throughout this paper, the alphabet has at least two elements.

\subsection{On the Powers of a Mealy Automaton and its Connected Components}

Let $\mathcal{M}=(A, \Sigma, \delta, \rho)$ be a Mealy automaton.

Considering the underlying graph of $\mathcal{M}$, it makes sense to look at its connected components. If $\mathcal{M}$ is reversible, its connected components are always strongly connected (its transition functions are permutations of a finite set).

A convenient and natural operation is to raise $\mathcal{M}$ to the power $n$, for some $n>0$ : its $n$-th power is the Mealy automaton

$$
\mathcal{M}^{n}=\left(A^{n}, \Sigma,\left(\delta_{i}: A^{n} \rightarrow A^{n}\right)_{i \in \Sigma},\left(\rho_{\mathbf{u}}: \Sigma \rightarrow \Sigma\right)_{\mathbf{u} \in A^{n}}\right) .
$$

If $\mathcal{M}$ is reversible, so is each of its powers.

If $\mathcal{M}$ is reversible, we can be more precise on the behavior of the connected components of its powers. As highlighted in [12], they have a very peculiar form: if $\mathcal{C}$ is a connected component of $\mathcal{M}^{n}$ for some $n$ and $\mathbf{u}$ is a state of $\mathcal{C}$, we obtain a connected component of $\mathcal{M}^{n+1}$ by choosing a state $x \in A$ and building the connected component of $\mathbf{u} x$, denote it by $\mathcal{D}$. For any state $\mathbf{v}$ of $\mathcal{C}$, there exists a state of $\mathcal{D}$ prefixed with $\mathbf{v}$ :

$$
\exists \mathbf{s} \in \Sigma^{*} \mid \delta_{\mathbf{s}}(\mathbf{u})=\mathbf{v} \quad \text { and } \quad \delta_{\mathbf{s}}(\mathbf{u} x)=\mathbf{v} \delta_{\rho_{\mathbf{u}}(\mathbf{s})}(x) .
$$

Furthermore, if $\mathbf{u} y$ is a state of $\mathcal{D}$, for some state $y \in A$ different from $x$, then $\delta_{\mathbf{s}}(\mathbf{u} x)$ and $\delta_{\mathbf{s}}(\mathbf{u} y)$ are two different states of $\mathcal{D}$ prefixed with $\mathbf{v}$, because of the reversibility of $\mathcal{M}^{n+1}$ : the transition function $\delta_{\rho_{\mathbf{u}}(\mathbf{s})}$ is a permutation.

Hence $\mathcal{D}$ can be seen as consisting of several full copies of $\mathcal{C}$ and $\# \mathcal{C}$ divides $\# \mathcal{D}$. They have the same size if and only if, once fixed some state $\mathbf{u}$ of $\mathcal{C}$, for any different states $x, y \in A, \mathbf{u} x$ and $\mathbf{u} y$ cannot both belong to $\mathcal{D}$. 
If all of those connected components of $\mathcal{M}^{n+1}$ built from $\mathcal{C}$ have the same size as $\mathcal{C}$, we say that $\mathcal{C}$ splits up totally. If all the connected components of an automaton split up totally, we say that the automaton splits up totally.

\section{$3 \quad$ A Maximal Family for Groups}

Antonenko and Russyev both investigated a family of Mealy automata such that the finiteness of the generated group (Russyev [17]) or semigroup (Antonenko [2]) is inherent to the structure of the automaton, regardless of its production functions. In fact, though they use different definitions and names, they study the same family: automata where every cycle is a sink component. Antonenko has proved that this family is maximal in the non-invertible case: if an automaton admits a cycle which is not a sink component, it can be enriched to generate an infinite semigroup.

To prove his result, Antonenko analyzes different cases and, in each situation, exhibits an element of infinite order in the semigroup. In this section we prove the maximality of the former family for groups, using completely different techniques. We adopt and adapt Russyev's nomenclature.

\subsection{How to Exit from a Cycle?}

Let $\mathcal{A}=(A, \Sigma, \delta)$ be an automaton. A cycle of length $n \in \mathbb{N}$ in the automaton $\mathcal{A}$ is a sequence of transitions of $\mathcal{A}$

$$
x_{1} \stackrel{i_{1}}{\longrightarrow} x_{2}, \quad \ldots, \quad x_{n-1} \stackrel{i_{n-1}}{\longrightarrow} x_{n}, \quad x_{n} \stackrel{i_{n}}{\longrightarrow} x_{1}
$$

where $x_{1}, \ldots, x_{n}$ are pairwise different states in $A$ and $i_{1}, \ldots, i_{n}$ are some letters of $\Sigma$.

The label of this cycle from the state $x_{k}$ is the word $i_{k} \cdots i_{n} i_{1} \cdots i_{k-1}$.

This cycle is with external exit if there exist $k$ with $1 \leq k \leq n$ and $i \in \Sigma$ satisfying $\delta_{i}\left(x_{k}\right) \notin\left\{x_{1}, \ldots, x_{n}\right\}$. It is with internal exit if there exist $k$ with $1 \leq k \leq n$ and $i \in \Sigma$ satisfying $\delta_{i}\left(x_{k}\right) \in\left\{x_{1}, \ldots, x_{n}\right\}$ and $\delta_{i}\left(x_{k}\right) \neq$ $\delta_{i_{k}}\left(x_{k}\right)$. We could say that a cycle is with exit without specifying the nature of the exit. In all other cases, this cycle is without exit. Examples are given in Fig. 3 .

Note that the existence of a cycle with internal exit induces the existence of a (possibly shorter) cycle with external exit. For example in Fig. 3, the cycle $1 \stackrel{a}{\rightarrow} 2 \stackrel{a}{\rightarrow} 3 \stackrel{a}{\rightarrow} 4 \stackrel{a}{\rightarrow} 1$ has two internal exits: $4 \stackrel{b}{\rightarrow} 4$ and $3 \stackrel{b}{\rightarrow} 1$; the 


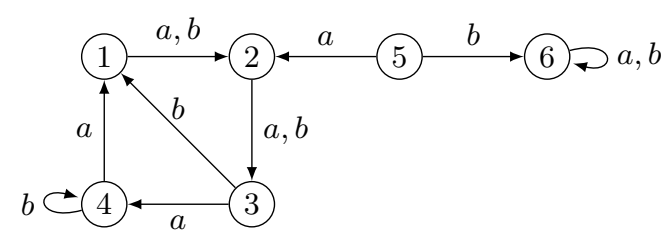

Fig. 3. The cycle $1 \stackrel{a}{\rightarrow} 2 \stackrel{a}{\rightarrow} 3 \stackrel{b}{\rightarrow} 1$ is with external exit; the cycle $1 \stackrel{a}{\rightarrow} 2 \stackrel{a}{\rightarrow} 3 \stackrel{a}{\rightarrow} 4 \stackrel{a}{\rightarrow} 1$ is with internal exit; and the cycle $6 \stackrel{a}{\rightarrow} 6$ is without exit.

first one leads to the cycle $4 \stackrel{b}{\rightarrow} 4$ with external exit, while the second one leads to the cycle $1 \stackrel{a}{\rightarrow} 2 \stackrel{a}{\rightarrow} 3 \stackrel{b}{\rightarrow} 1$ with external exit.

Proposition 1 ([2, 17]). Whenever an automaton $\mathcal{A}$ admits no cycle with exit, whatever choice is maid for the production functions $\rho$, the enriched automaton $(\mathcal{A}, \rho)$ generates a finite (semi)group.

\subsection{A Pumping Lemma for the Reversible Two-State Automata}

It is proved in [12, Lemma 10] that in the case of a reversible Mealy automaton $\mathcal{M}$ with exactly two states, if some power of $\mathcal{M}$ splits up totally, then all the later powers of $\mathcal{M}$ split up totally. We can deduce the following result which can be seen as a pumping lemma: if the generated semigroup is infinite, sufficiently long paths can be considered in the dual automaton to turn indefinitely in a cycle.

Lemma 2 (Pumping Lemma). Let $\mathcal{M}$ be a reversible Mealy automaton with two states $\{x, y\}$. The automaton $\mathcal{M}$ generates an infinite semigroup if and only if, for any integer $N \in \mathbb{N}$, there exists a word $\mathbf{u} \in\{x, y\}^{*}$ of length at least $N$ such that the states $\mathbf{u} x$ and $\mathbf{u} y$ belong to the same connected component of $\mathcal{M}^{|\mathbf{u}|+1}$.

\subsection{The Family of Automata with no Cycles with Exit is Maximal for Groups}

We prove here that any automaton which admits a cycle with exit can be enriched in order to generate an infinite group. We analyze several simple cases in Lemmas 3, 4, and 5 which contribute to prove the general case of Theorem 6 . 
Lemma 3. Any automaton over a binary alphabet with a cycle with external exit can be enriched to generate an infinite group.

Proof. Let $\mathcal{A}$ be an automaton over a binary alphabet $\{0,1\}$ with a cycle $\mathcal{C}$ with external exit as shown in Fig. 4.

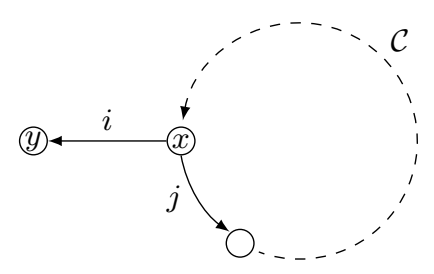

Fig. 4. The cycle $\mathcal{C}$ is with external exit: $y=\delta_{i}(x), y \notin \mathcal{C}$.

Take the following permutations on the alphabet : $\rho_{y}$ permutes the letters of the alphabet and $\rho_{z}$ stabilizes the alphabet for any other state $z$ (in particular for any state of $\mathcal{C}$ ).

Let $\mathbf{s} \in\{0,1\}^{+}$be the label of $\mathcal{C}$ from $x$. For any $n \in \mathbb{N}$, the words $\mathbf{s}^{n} i 0$ and $\mathbf{s}^{n} i 1$ belong to a same connected component of $\mathfrak{d}(\mathcal{A}, \rho)^{|\mathbf{s}|+2}: \rho_{x}\left(\mathbf{s}^{n} i 0\right)=$ $\mathbf{s}^{n} i 1$. The Mealy automaton $\mathfrak{d}(\mathcal{A}, \rho)$ is reversible and has two states, so we can apply the Pumping Lemma and conclude on the infiniteness of $\langle(\mathcal{A}, \rho)\rangle$ by $(\mathbf{F} 2)$ and $(\mathbf{F} 3)$.

Lemma 4 (River of no return Lemma). Let $\mathcal{A}$ be an automaton and $\mathcal{C}$ a cycle of $\mathcal{A}$. If $\mathcal{C}$ admits an external exit to some state and is not reachable from this state, then $\mathcal{A}$ can be enriched to generate an infinite group.

Proof. The idea of this proof is to mimic the adding machine (see Fig. 1). Again, Fig. 4 illustrates the situation: $\mathcal{C}$ admits an external exit to the state $y$, the additional hypothesis being that $\mathcal{C}$ is not reachable from $y$ (and the alphabet is not supposed binary any longer).

Denote the label of $\mathcal{C}$ from $x$ by $\mathbf{s}=j \mathbf{t}$ with $j \in \Sigma$ and $\mathbf{t} \in \Sigma^{*}$. We choose the following production functions on the alphabet: $\rho_{x}$ is the transposition of $i$ and $j$ and $\rho_{z}$ is the identity for any other state $z$.

As for the adding machine, the orbit of $(j \mathbf{t})^{n}$ under the action of $\rho_{x}$ has size $2^{n}$. Therefore the element $x$ is of infinite order and so is the group $\langle(\mathcal{A}, \rho)\rangle$.

Lemma 5. Any reversible automaton with a cycle with exit can be enriched to generate an infinite group. 
Proof. Let $\mathcal{A}=(A, \Sigma, \delta)$ be a reversible automaton with a cycle with exit.

As $\mathcal{A}$ is reversible, it admits some states $x, y, z$ with $x \neq y$ such that there exist a transition from $x$ to $z$ and a transition from $y$ to $z$. We can choose the permutations $\rho_{x}$ and $\rho_{y}$ such that these transitions have the same output and take identity for all the other permutations.

The enriched automaton $(\mathcal{A}, \rho)$ is invertible and reversible but not bireversible. Hence it generates an infinite group by $(\mathbf{F 4})$.

The next theorem is the main result of this paper.

Theorem 6. Any automaton with a cycle with exit can be enriched into an invertible Mealy automaton generating an infinite group.

Proof. Let $\mathcal{A}=(A, \Sigma, \delta)$ be an automaton with a cycle with exit.

By (F1), we can suppose, without loss of generality, that $\mathcal{A}$ is pruned. If there exists a transition not belonging to a cycle, as the starting state of this transition is reachable from a cycle, Lemma 4 applies and we are done.

We can assume now that any transition belongs to (at least) one cycle. If $\mathcal{A}$ is reversible, it can be enriched to generate an infinite group by Lemma 5.

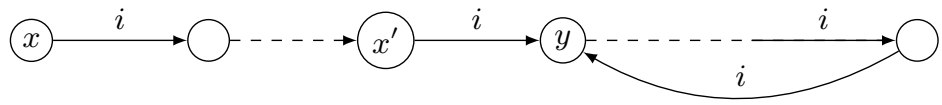

Fig. 5. Path from $x$ with all transitions labelled by $i$.

We can suppose now that $\mathcal{A}$ is not reversible: there exist a state $x$ and a letter $i$ such that $x$ has no incoming transition labelled by $i$. Consider the path starting at $x$ with all transitions labelled by $i$ as shown in Fig. 5 . This path loops on some state $y \neq x$. Denote by $\mathcal{C}$ the resulting cycle. We

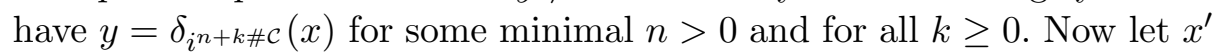
denote the state $\delta_{i^{n-1}}(x)$ : we have $x^{\prime} \notin \mathcal{C}$.

The transition $x^{\prime} \stackrel{i}{\longrightarrow} y$ belongs to some cycle by hypothesis and this cycle is not $\mathcal{C}$ by construction. Therefore $\mathcal{C}$ admits an external exit $y^{\prime} \stackrel{j}{\longrightarrow} y^{\prime \prime}$ (with $j \neq i$ ). Hence $x^{\prime}$ is reachable from $y$ and so from $\mathcal{C}$, by hypothesis, but does not belong to $\mathcal{C}$, by construction. The automaton $\mathcal{B}=$ $\left(A,\{i, j\},\left(\delta_{i}, \delta_{j}\right)\right)$ contains the cycle $\mathcal{C}$ and the transition $y^{\prime} \stackrel{j}{\longrightarrow} y^{\prime \prime}$. So $\mathcal{B}$ 
can be enriched to generate an infinite group by Lemma 3, say with $\rho=$ $\left(\rho_{z}:\{i, j\} \rightarrow\{i, j\}\right)_{z \in A}$. This group is a quotient of any group obtained by completing each $\rho_{z}$ from $\{i, j\}$ into $\Sigma$, and we can conclude.

\section{Acknowledgments}

The authors would like to thank Jean Mairesse who has detected a serious gap in a previous version of this paper.

\section{References}

1. Akhavi, A., Klimann, I., Lombardy, S., Mairesse, J., Picantin, M.: On the finiteness problem for automaton (semi)groups. Int. J. Algebra Comput. 22(6) (2012) 26p.

2. Antonenko, A.S.: On transition functions of Mealy automata of finite growth. Matematychni Studii. 29(1) (2008) 3-17

3. Antonenko, A.S., Berkovich, E.L.: Groups and semigroups defined by some classes of Mealy automata. Acta Cybernetica 18(1) (2007) 23-46

4. Bartholdi, L., Silva, P.: Groups defined by automata. arXiv:cs.FL/1012.1531.

5. Bondarenko, I., Bondarenko, N., Sidki, S., Zapata, F.: On the conjugacy problem for finite-state automorphisms of regular rooted trees (with an appendix by Raphaël M. Jungers). Groups Geom. Dyn. 7(2) (2013) 323-355

6. Cain, A.: Automaton semigroups. Theor. Comput. Sci. 410(47-49) (2009) 50225038

7. De Felice, S., Nicaud, C.: Random generation of acyclic deterministic automata using the recursive method. In: Proc. 8th CSR. Volume 7913 of Lecture Notes in Computer Science. (2013) 88-99

8. Dixon, J.D.: The probability of generating the symmetric group. Math. Z. 110 (1969) 199-205

9. Gillibert, P.: The finiteness problem for automaton semigroups is undecidable. arXiv:cs.FL/1304.2295 (2013)

10. Grigorchuk, R., Nekrashevich, V., Sushchanskiı̌, V.: Automata, dynamical systems, and groups. Tr. Mat. Inst. Steklova 231 (2000) 134-214

11. Jaikin-Zapirain, A., Pyber, L.: Random generation of finite and profinite groups and group enumeration. Ann. of Math. (2) 173(2) (2011) 769-814

12. Klimann, I.: The finiteness of a group generated by a 2-letter invertible-reversible Mealy automaton is decidable. In: Proc. 30th STACS. Volume 20 of LIPIcs. (2013) $502-513$

13. Klimann, I., Mairesse, J., Picantin, M.: Implementing computations in automaton (semi)groups. In: Proc. 17th CIAA. Volume 7381 of LNCS. (2012) 240-252

14. Maltcev, V.: Cayley automaton semigroups. Int. J. Algebra Comput. 19(1) (2009) $79-95$

15. Mintz, A.: On the Cayley semigroup of a finite aperiodic semigroup. Int. J. Algebra Comput. 19(6) (2009) 723-746

16. Nekrashevych, V.: Self-similar groups. Volume 117 of Mathematical Surveys and Monographs. American Mathematical Society, Providence, RI (2005)

17. Russyev, A.: Finite groups as groups of automata with no cycles with exit. Algebra and Discrete Mathematics 9(1) (2010) 86-102 
18. Savchuk, D., Vorobets, Y.: Automata generating free products of groups of order 2. J. Algebra 336(1) (2011) 53-66

19. Sidki, S.: Automorphisms of one-rooted trees: growth, circuit structure, and acyclicity. J. Math. Sci. (New York) 100(1) (2000) 1925-1943 Algebra, 12.

20. Silva, P., Steinberg, B.: On a class of automata groups generalizing lamplighter groups. Int. J. Algebra Comput. 15(5-6) (2005) 1213-1234

21. Steinberg, B., Vorobets, M., Vorobets, Y.: Automata over a binary alphabet generating free groups of even rank. Int. J. Algebra Comput. 21(1-2) (2011) 329-354

22. Šunik, Z., Ventura, E.: The conjugacy problem in automaton groups is not solvable. Journal of Algebra 364(0) (2012) 148 - 154 